\title{
A Stable and Accurate Davies-like Relaxation Procedure using Multiple Penalty Terms for Lateral Boundary Conditions
}

\author{
Hannes Frenander \\ Division of Computational Mathematics, Department of Mathematics, Linköping \\ University, SE-58183 Linköping, Sweden.e-mail: hannes.frenander@liu.se \\ Jan Nordström \\ Division of Computational Mathematics, Department of Mathematics, Linköping \\ University, SE-58183 Linköping, Sweden. e-mail: jan.nordstrom@liu.se
}

\begin{abstract}
A lateral boundary treatment using summation-by-parts operators and simultaneous approximation terms is introduced. The method is similar to Davies relaxation technique used in the weather prediction community and have similar areas of application, but is also provably stable. In this paper, it is shown how this technique can be applied to the shallow water equations, and that it reduces the errors in the computational domain. [

Keywords: Boundary conditions, Boundary layers, Summation-by-parts, Energy method, Convergence, Shallow water equations
\end{abstract}

\section{Introduction}

Accurate numerical calculations on large domains are often unfeasible. ${ }_{3}$ To reduce the computational effort, a coarse global mesh is often used with

Abbreviations:

Summation-by-parts (SBP)

Simultaneous approximations terms (SAT)

Multiple penalty technique (MPT) 
4 int

inter-spaced local domains. The local domains are typically employed where fine meshes are needed to capture phenomena with higher accuracy than in the global domain. In the weather prediction community, local domains are often used to model local weather phenomena.

The local domains need to be matched correctly to the global one. Davies (1976) introduced a lateral boundary procedure for weather prediction models where the numerical results on the coarse grid are interpolated into the local, fine grid domain. The method was later referred to as Davies relaxation. Other types of interpolation methods are also used within the weather prediction community, see Williamson and Browning (1974), Birchfield (1960) and Hill (1968). In most applications, one aims for accurate results in the local domains, while the accuracy in the global domain is considered given. Therefore, the coupling between the domains is in most cases neglected. There are methods, see Harrison and Elsberry (1972), where the coupling between the meshes is considered, such that the results from the local domain influence the global one. However, this coupling often introduces stability issues unless optimally done, see for example Nordström et al. (2009b), Nordström et al. (2009a). In this work, we will only consider the case where the domains are uncoupled, which is known as one-way nesting.

Many existing lateral boundary schemes produce reflections at the lateral boundaries and experience issues due to over-specification of boundary data (Davies (1983)). These issues are commonly dealt with by choosing the relaxation coefficients properly (Lehmann (1993)) and adding dissipation to the numerical scheme (Davies (1983)). However, altering the scheme in these manners may result in an inaccurate and even unstable scheme. The method introduced in this work can effectively reduce reflections at the lateral boundaries (Nordström et al. (2014)), without ruining stability and accuracy.

We demonstrate our technique on the shallow water equations, discretized using operators with the summation-by-parts (SBP) property (Carpenter et al. (1999), Strand (1994), Mattsson and Nordström (2004)), augmented with simultaneous approximation terms (SAT) (Carpenter et al. (1994)). This is often referred to as the SBP-SAT technique, and a comprehensive review is given by Svärd and Nordström (2014). If well-posed boundary conditions are available, this technique yields stable numerical schemes (Carpenter et al. (1999)).

Nordström et al. (2014) showed that if data is available inside the computational domain, additional penalty terms can be applied without ruining 
stability. This method, which we call the multiple penalty technique (MPT), can be used to assimilate global data into local area models, construct nonreflecting boundaries, improve accuracy of the scheme and increase the rate of convergence to steady state, see also Erickson and Nordström (2014). The MPT is similar to the Davies relaxation technique mentioned above, but can also be proven to be stable.

The rest of the paper will proceed as follows. In the following section, we define well-posedness of a problem and stability of a numerical scheme. Section 3 describes the SBP-SAT technique and MPT on a simple model problem in one space dimension. In section 4, we derive well-posed boundary conditions for the shallow water equations and discretize them by using the SBP-SAT technique. Stability conditions are derived and the MPT is applied such that stability is preserved. In section 5, numerical experiments are performed to illustrate the increased rate of convergence when using the MPT. The assessments of this paper are concluded in section 6 .

\section{Preliminaries}

Before moving on to the problem under consideration, we will define well-posedness of an initial-boundary value problem (IBVP) and stability of a numerical approximation.

Consider the problem

$$
\begin{aligned}
\frac{\partial u}{\partial t} & =H u+F, \quad \overline{\mathbf{r}} \in \Omega, t \geq 0 \\
u(\overline{\mathbf{r}}, 0) & =f(\overline{\mathbf{r}}) \\
L u(\overline{\mathbf{r}}, t) & =g(t), \quad \overline{\mathbf{r}} \in \delta \Omega
\end{aligned}
$$

where $H$ is a differential operator, $\Omega$ the spatial domain, $\delta \Omega$ the boundary to $\Omega, \overline{\mathbf{r}}$ the position vector and $F, f, g$ are known functions. In (1), $L$ is a boundary operator. The problem (1) is well-posed if it has a unique solution that, for $g=F=0$, satisfies

$$
\|u(\overline{\mathbf{r}}, t)\|^{2} \leq K_{c}^{1}\|f\|^{2}
$$

where $K_{c}^{1}$ is bounded for finite time and independent of $f$. Moreover, (1) is strongly well-posed if

$$
\|u(\overline{\mathbf{r}}, t)\|^{2} \leq K_{c}^{2}\left[\|f\|^{2}+\int_{0}^{t}\left(\|F\|^{2}+\|g\|^{2}\right) d \tau\right]
$$


for non-zero $g$ and $F$. In (2) and (3), $\|f\|,\|g\|$ and $\|F\|$ can be expressed in arbitrary norms. The function $K_{c}^{2}$ is bounded for finite time and does not depend on $f, g$ and $F$.

Let a global semi-discrete approximation of (1) be

$$
\begin{aligned}
\frac{\partial \mathbf{v}}{\partial t} & =\mathbf{H} \mathbf{v}+\mathbf{F}, \quad t \geq 0 \\
\mathbf{v}(0) & =\mathbf{f} \\
\mathbf{L v}(t) & =\mathbf{g}
\end{aligned}
$$

where $\mathbf{v}$ is the numerical approximation of $u$ and $\mathbf{H}$ is a discrete operator that approximates $H$. The functions $\mathbf{F}, \mathbf{f}, \mathbf{g}$ are grid functions of $F, f$ and $g$, i.e. the values are injected at the grid points. Analogously to (2), (4) is stable if

$$
\|\mathbf{v}(t)\|^{2} \leq K_{d}^{1}\|\mathbf{f}\|^{2}
$$

for $\mathbf{F}=\mathbf{g}=0$. In (5), $K_{d}^{1}$ is bounded for finite time and independent of $\mathbf{f}$ and the mesh size. For non-zero $\mathbf{g}$ and $\mathbf{F}$, we say that (4) is strongly stable if

$$
\|\mathbf{v}(t)\|^{2} \leq K_{d}^{2}\left[\|\mathbf{f}\|^{2}+\int_{0}^{t}\left(\|\mathbf{F}\|^{2}+\|\mathbf{g}\|^{2}\right) d \tau\right]
$$

where $K_{d}^{2}$ is bounded for finite time and independent of $\mathbf{f}, \mathbf{g}, \mathbf{F}$ and the meshsize. As in the continuous estimate, $\|\mathbf{f}\|,\|\mathbf{g}\|$ and $\|\mathbf{F}\|$ may be given in arbitrary norms. For a detailed discussion of well-posedness and stability, the reader is referred to Svärd and Nordström (2014).

\section{The SBP-SAT-MPT technique on a model problem}

We start by illustrating the SBP-SAT-MPT technique on the advection equation,

$$
u_{t}+a u_{x}=0 .
$$

In (7), $a>0$ is a constant and the domain under consideration is $x \in$ $[0,1], t \geq 0$. Unless stated otherwise, we use subscripts to denote partial derivatives, i.e. $u_{x}=\partial u / \partial x$ and $u_{t}=\partial u / \partial t$ in (7).

For determining well-posed boundary conditions to (7), we multiply with $u$ and integrate over the spatial domain,

$$
\int_{0}^{1} u u_{t} d x+a \int_{0}^{1} u u_{x} d x=\frac{1}{2} \int_{0}^{1}\left(u^{2}\right)_{t} d x+\frac{a}{2} \int_{0}^{1}\left(u^{2}\right)_{x} d x=0 .
$$


By defining the standard $L_{2}$-norm $\|u\|=\sqrt{\int_{0}^{1} u^{2} d x}$ and imposing the boundary condition $u(0, t)=g(t)$, where $g(t)$ is known boundary data, (8) becomes

$$
\left(\|u\|^{2}\right)_{t}=a g^{2}(t)-a u^{2}(1, t) .
$$

After integrating (9) in time, we obtain

$$
\|u\|^{2}+a \int_{0}^{t} u^{2}(1, \tau) d \tau=\|f\|^{2}+a \int_{0}^{t} g^{2}(\tau) d \tau .
$$

Hence, one can conclude that $\|u\|$ satisfy an estimate of the form (3), and (7) with the boundary condition $u(0, t)=g(t)$ is therefore strongly well-posed.

\subsection{The semi-discrete problem}

Equation (7) is discretized in space using the SBP finite difference operator $\mathbf{D}=\mathbf{P}^{-1} \mathbf{Q}$ :

$$
\mathbf{v}_{t}+a \mathbf{P}^{-\mathbf{1}} \mathbf{Q} \mathbf{v}=\alpha_{0} \mathbf{P}^{-\mathbf{1}}\left(v_{0}-g(t)\right) \tilde{\mathbf{e}}_{0},
$$

where $\tilde{\mathbf{e}}_{0}=(1,0, \ldots, 0), \alpha_{0}$ is a penalty coefficient to be determined and $\mathbf{v}$ is the numerical approximation of $u$. The matrix $\mathbf{P}$ is symmetric and positive definite, and the matrix $\mathbf{Q}$ satisfies the SBP property: $\mathbf{Q}+\mathbf{Q}^{T}=$ $\operatorname{diag}(-1,0, \ldots, 0,1)$. The SAT-term on the right-hand side of (10) enforces the boundary condition $u(0, t)=g(t)$ weakly; see Carpenter et al. (1994) for more details of this technique.

By multiplying 10$)$ with $\mathbf{v}^{T} \mathbf{P}$ from the left, one obtains

$$
\mathbf{v}^{T} \mathbf{P} \mathbf{v}_{t}+a \mathbf{v}^{T} \mathbf{Q} \mathbf{v}=\alpha_{0} v_{0}\left(v_{0}-g(t)\right) .
$$

The multiplication with $\mathbf{v}^{T} \mathbf{P}$ from the left is analogous to multiplication of $u$ and integration over the domain in the continuous setting. Adding the transpose of (11) to itself and using the SBP property of $\mathbf{Q}$ results in

$$
\left(\|\mathbf{v}\|_{\mathbf{P}}^{2}\right)_{t}=a g^{2}(t)-a v_{N}^{2}-a\left(v_{0}-g(t)\right)^{2}
$$

where we have chosen $\alpha_{0}=-a$ and defined $\|\mathbf{v}\|_{\mathbf{P}}{ }^{2}=\mathbf{v}^{T} \mathbf{P} \mathbf{v}$. After integrating (12) in time, one can clearly see that $\|\mathbf{v}\|$ satisfies an estimate of the form $(6)$, and $(10)$ is therefore strongly stable. Note also that $(12)$ mimics $(9)$ if $v_{0}=g(t)$.

As the boundary condition is imposed weakly with the SAT, i.e. $v_{0}$ is allowed to slightly deviate from $g(t)$, additional damping is introduced that stabilizes the numerical scheme. For more details of the SBP finite difference operators, the reader is referred to Strand (1994) and Svärd and Nordström (2014). 


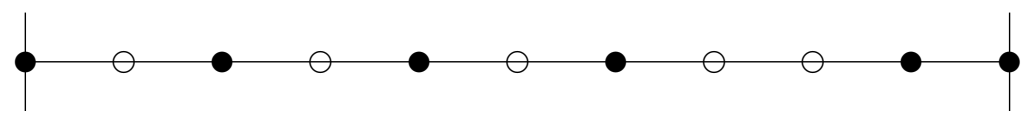

Figure 1: Illustration of the mesh in one space dimension. The circles denotes the grid points and filled circles denotes grid points where SAT's are applied.

\subsection{The multiple penalty technique}

Consider the problem (7) and assume that data can be obtained at several grid points anywhere in the domain, see Figure 1 for an illustration. We denote the set of grid points where additional data is known as $\Omega_{M}$.

By applying penalty terms (SAT's) wherever data is available, (10) can be extended to

$$
\begin{aligned}
\mathbf{v}_{t}+a \mathbf{P}^{-1} \mathbf{Q} \mathbf{v} & =\alpha_{0} \mathbf{P}^{-1}\left(v_{0}-g(t)\right) \tilde{\mathbf{e}}_{0} \\
& +\sum_{i \in \Omega_{M}} \alpha_{i} \mathbf{P}^{-1}\left(v_{i}-g_{i}(t)\right) \tilde{\mathbf{e}}_{i}
\end{aligned}
$$

In (13), $\alpha_{i}$ are additional penalty coefficients to be determined, $g_{i}(t)$ are known data, and $\tilde{\mathbf{e}}_{i}$ are projection vectors at grid point $i$. That is, all elements of $\tilde{\mathbf{e}}_{i}$ are zero, except the element $i$, which is one. As an example, consider $\tilde{\mathbf{e}}_{3}=[0,0,1,0, \ldots, 0]^{T}$. The additional SAT's in $(13)$ are referred to as multiple penalty terms.

Stability is obtained in the same way as in section 3.1, i.e. 13 is multiplied with $\mathbf{v}^{\mathbf{T}} \mathbf{P}$ from the left, and the transpose of the outcome is added to the expression,

$$
\begin{aligned}
\left(\|\mathbf{v}\|_{\mathbf{P}}{ }^{2}\right)_{t} & =a g^{2}(t)-a\left(v_{0}-g(t)\right)^{2}-a v_{N}^{2} \\
& +2 \sum_{i \in \Omega_{M}} \alpha_{i} v_{i}\left(v_{i}-g_{i}(t)\right)
\end{aligned}
$$

where we have used $\alpha_{0}=-a$. Equation (14) can be rearranged into

$$
\begin{aligned}
\left(\|\mathbf{v}\|_{\mathbf{P}}^{2}\right)_{t} & =a g^{2}(t)-a\left(v_{0}-g(t)\right)^{2}-a v_{N}^{2} \\
& +\sum_{i \in \Omega_{M}} \alpha_{i}\left(v_{i}-g_{i}\right)^{2}+\alpha_{i}\left(v_{i}^{2}-g_{i}^{2}\right) .
\end{aligned}
$$

After integrating (15) in time, one can observe that $\|\mathbf{v}\|$ satisfies an estimate of the form (6) if $\alpha_{i} \leq 0$, and the scheme is therefore strongly stable for these values of $\alpha_{i}$. Note also that if $g_{i}=v_{i}$, 15ill mimic the continuous 
estimate (9). The parameters $\alpha_{i}$ can be used to alter the scheme in different ways (while maintaining stability). For example, one can use the MPT to construct buffer zones to reduce reflections, increase the order of accuracy or, as illustrated also in this paper, increase the rate of convergence, see Nordström et al. (2014).

\subsection{A numerical example}

Consider a slightly altered version of (7),

$$
\begin{aligned}
u_{t}+a u_{x} & =F(x, t) \\
u(0, t) & =g(t) \\
u(x, 0) & =0
\end{aligned}
$$

where $F(x, t)$ is a known forcing function and $a=1$. By choosing $F(x, t)=$ $-2 \pi \sin (2 \pi x)$ and $g(t)=1$, the exact steady-state solution to (16) becomes $u_{S S}(x)=\cos (2 \pi x)$. This technique is known as the method of manufactured solutions (Lindström and Nordström (2010)). In this test case, we assume that we know the solution in the domains $x \in[0,0.2]$ and $x \in[0.8,1]$. We can then apply the MPT by setting the data $g_{i}=u_{S S}\left(x_{i}\right)$ at the corresponding grid points. The aim is to get a better approximation in the region $x \in$ $[0.2,0.8]$, where the solution is unknown.

We now employ the SBP-SAT technique described above to (16) and measure the deviation from the steady-state solution, $u_{S S}$. Additional penalty terms are applied in the domains $x \in[0,0.2]$ and $x \in[0.8,1]$ and the number of grid points is $N=40$. For simplicity, we choose $\alpha_{i}=-1$. A fourth order explicit Runge Kutta is used to integrate in time and the error at the final time $T=5$ is calculated as $\|\mathbf{e}\|_{\mathbf{P}}{ }^{2}=\left(\mathbf{v}-\mathbf{u}_{\mathbf{S S}}\right)^{T} \mathbf{P}\left(\mathbf{v}-\mathbf{u}_{\mathbf{S S}}\right)$. However, the following results would be similar in any $L_{2}$-equivalent norm. As illustrated in Figure 2, the error decrease as the MPT is applied, also at grid points in the domain $x \in[0.2,0.8]$, where no data is available.

\section{The shallow water equations}

Consider the inviscid shallow water equations,

$$
\begin{array}{r}
\overline{\mathbf{V}}_{t}+\overline{\mathbf{V}} \nabla \cdot \overline{\mathbf{V}}+f \mathbf{e}_{3} \times \overline{\mathbf{V}}+g \nabla h=0 \\
h_{t}+\overline{\mathbf{V}} \cdot \nabla h+h \nabla \cdot \overline{\mathbf{V}}=0
\end{array}
$$




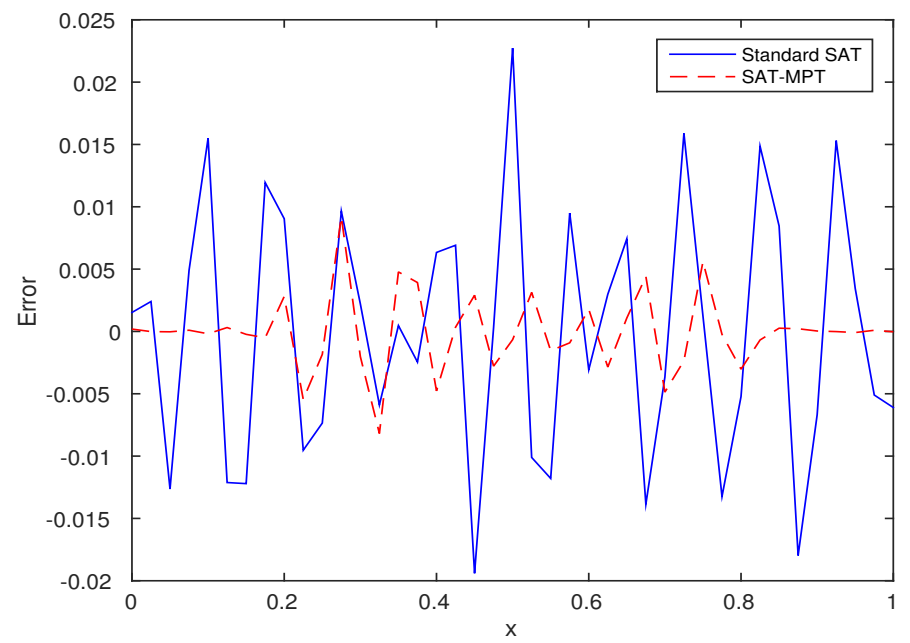

Figure 2: The error at $T=5$ when using standard and multiple penalties. $N=40$

where $\overline{\mathbf{V}}$ is the velocity vector, $f$ the Coriolis parameter, $h$ the height and $g$ the gravitational constant. The vector $\mathbf{e}_{3}=[0,0,1]^{T}$ is the unit vector in the $z$-direction. In this work, we consider (17) linearized around a reference state $\mathbf{u}_{0}=[\bar{u}, \bar{v}, H]^{T}$, in which $H$ is the reference height and $\bar{u}, \bar{v}$ reference state velocities. More details about linearizing and symmetrizing the shallow water equations is provided by Ghader and Nordström (2014).

Consider the linearized and symmetrized problem

$$
\begin{aligned}
\mathbf{u}_{t}+\mathbf{A} \mathbf{u}_{x}+\mathbf{B u}_{y}+\mathbf{C u} & =\mathbf{F}(x, y, t) \\
\mathbf{L}_{\mathbf{w}} \mathbf{u}(0, y, t) & =\mathbf{g}_{\mathbf{w}}(y, t) \\
\mathbf{L}_{\mathbf{e}} \mathbf{u}(1, y, t) & =\mathbf{g}_{\mathbf{e}}(y, t) \\
\mathbf{L}_{\mathbf{s}} \mathbf{u}(x, 0, t) & =\mathbf{g}_{\mathbf{s}}(x, t) \\
\mathbf{L}_{\mathbf{n}} \mathbf{u}(x, 1, t) & =\mathbf{g}_{\mathbf{n}}(x, t) \\
\mathbf{u}(x, y, 0) & =\mathbf{f}(x, y),
\end{aligned}
$$

where the domain under consideration is $x, y \in[0,1], t \geq 0$. This domain constitutes a local domain inserted in a global one, and the boundary data has been obtained from calculations using the global mesh, see Figure 3 for 


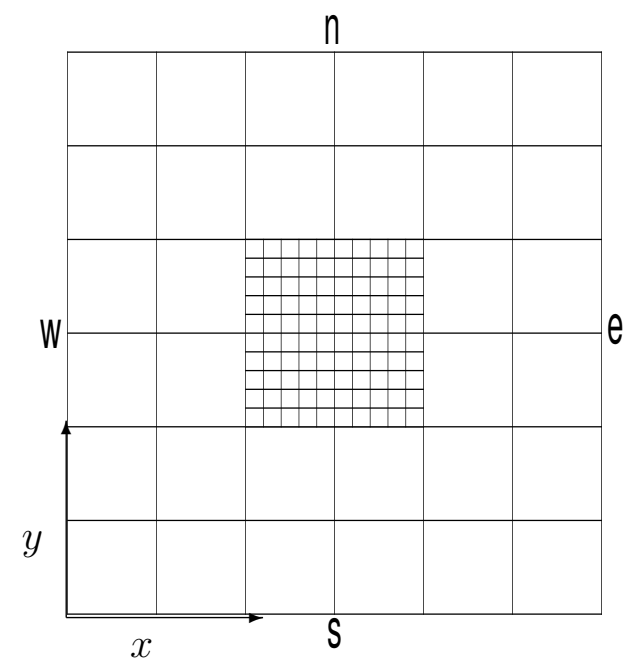

Figure 3: An illustration of the problem set-up. The calculations are performed in the central domain where boundary data has been interpolated from the coarse, global domain. The notations $w, e, n, s$ refers to the west, east, north and south boundary, respectively. The central domain has its corners at $(0,0),(0,1),(1,0)$ and $(1,1)$.

174

an illustration. The solution to $(18)$ is

$$
\mathbf{u}=\left[u^{\prime}, v^{\prime}, \frac{g h^{\prime}}{\bar{c}}\right]^{T}
$$

where $g$ is the gravitational constant and $u^{\prime}, v^{\prime}$ are the deviation from $\bar{u}, \bar{v}$, respectively. The variable $h^{\prime}$ denotes the deviation from $H$, and $\mathbf{g}_{\mathbf{w}, \mathbf{e}, \mathbf{s}, \mathbf{n}}, \mathbf{f}$ are given boundary and initial data. For completeness, a forcing function $\mathbf{F}(x, y, t)$ is added to the right-hand side. In 18,

$$
\mathbf{A}=\left[\begin{array}{ccc}
\bar{u} & 0 & \bar{c} \\
0 & \bar{u} & 0 \\
\bar{c} & 0 & \bar{u}
\end{array}\right], \quad \mathbf{B}=\left[\begin{array}{ccc}
\bar{v} & 0 & 0 \\
0 & \bar{v} & \bar{c} \\
0 & \bar{c} & \bar{v}
\end{array}\right], \quad \mathbf{C}=\left[\begin{array}{ccc}
0 & -f & 0 \\
f & 0 & 0 \\
0 & 0 & 0
\end{array}\right]
$$

where $\bar{c}=\sqrt{g H}$ is the gravity wave speed and the matrix $\mathbf{C}$ describes the Coriolis force acting on the system. In this work, we focus on sub-critical flows, $\bar{u}^{2}+\bar{v}^{2}<\bar{c}^{2}$. 


\subsection{Well-posedness}

The boundary operators $\mathbf{L}_{\mathbf{w}, \mathbf{e}, \mathbf{s}, \mathbf{n}}$ must be determined such that 18 is well-posed. For clarity, the forcing function $\mathbf{F}(x, y, t)$ is neglected in the following analysis.

The technique in section 3.1 is applied to problem (18); that is, 180 is multiplied with $\mathbf{u}^{T}$ from the left and integrated over the spatial domain. One obtains,

$$
\left(\|\mathbf{u}\|^{2}\right)_{t}=-\int_{\partial \Omega} \mathbf{u}^{T}(\alpha \mathbf{A}+\beta \mathbf{B}) \mathbf{u} d s,
$$

where we have employed Greens theorem and defined $\|\mathbf{u}\|^{2}=\int_{\Omega} \mathbf{u}^{T} \mathbf{u} d \Omega$. In (19), $\hat{\mathbf{n}}=[d y,-d x]^{T} / \sqrt{d x^{2}+d y^{2}}=[\alpha, \beta]$ is the outward pointing normal to the domain. The term $d s=\sqrt{d x^{2}+d y^{2}}$ is the differential along the boundary $\partial \Omega$ to the domain $\Omega$. Since $\mathbf{C}$ is skew-symmetric, the Coriolis force does not affect the estimate (19).

By considering the domain shown in Figure 3, (19) can be written out explicitly for each separate part of the boundary,

$$
\begin{aligned}
\|\mathbf{u}\|_{t}^{2} & =-\oint_{\partial \Omega}\left[\mathbf{u}^{T} \mathbf{A} \mathbf{u}, \mathbf{u}^{T} \mathbf{B} \mathbf{u}\right] \cdot \hat{\mathbf{n}} d s \quad=\int_{x=0}^{1} \mathbf{u}(x, 0, t)^{T} \mathbf{B} \mathbf{u}(x, 0, t) d x \\
& -\int_{x=0}^{1} \mathbf{u}(x, 1, t)^{T} \mathbf{B} \mathbf{u}(x, 1, t) d x \quad+\int_{y=0}^{1} \mathbf{u}(0, y, t)^{T} \mathbf{A} \mathbf{u}(0, y, t) d y \\
& -\int_{y=0}^{1} \mathbf{u}(1, y, t)^{T} \mathbf{A} \mathbf{u}(1, y, t) d y .
\end{aligned}
$$

All boundary operators that bound the right-hand side of (20) result in an energy estimate, and well-posedness follows if the correct number of boundary conditions is used (Svärd and Nordström (2014)).

The boundary operators used in this work are

$$
\begin{aligned}
\mathbf{L}_{\mathbf{w}} & =\mathbf{S}_{\mathbf{A}} \boldsymbol{\Lambda}_{A}^{+} \mathbf{S}_{\mathbf{A}}^{T}=\mathbf{A}^{+} \\
\mathbf{L}_{\mathbf{s}} & =\mathbf{S}_{\mathbf{B}} \Lambda_{B}^{+} \mathbf{S}_{\mathbf{B}}^{T}=\mathbf{B}^{+} \\
\mathbf{L}_{\mathbf{e}} & =\mathbf{S}_{\mathbf{A}} \boldsymbol{\Lambda}_{A}^{-} \mathbf{S}_{\mathbf{A}}^{T}=\mathbf{A}^{-} \\
\mathbf{L}_{\mathbf{n}} & =\mathbf{S}_{\mathbf{B}} \boldsymbol{\Lambda}_{B}^{-} \mathbf{S}_{\mathbf{B}}^{T}=\mathbf{B}^{-},
\end{aligned}
$$

and $\boldsymbol{\Lambda}_{A, B}^{ \pm}$are the positive and negative part of the eigenvalue matrices, re- 
spectively. The matrices

$$
\mathbf{S}_{\mathbf{A}}=\frac{1}{\sqrt{2}}\left[\begin{array}{ccc}
0 & 1 & 1 \\
\sqrt{2} & 0 & 0 \\
0 & 1 & -1
\end{array}\right], \quad \mathbf{S}_{\mathbf{B}}=\frac{1}{\sqrt{2}}\left[\begin{array}{ccc}
\sqrt{2} & 0 & 0 \\
0 & 1 & 1 \\
0 & 1 & -1
\end{array}\right]
$$

are the similarity transformations that diagonalizes $\mathbf{A}, \mathbf{B}$. We can of course handle any type of flow field, but to exemplify we consider the case $\bar{u}>, \bar{v}>0$ and $\bar{u}^{2}+\bar{v}^{2}<\bar{c}^{2}$. Under these circumstances, the operators in (21) are

$$
\begin{aligned}
\mathbf{L}_{\mathbf{w}}=\mathbf{A}^{+}=\frac{1}{2}\left[\begin{array}{ccc}
\bar{u}+\bar{c} & 0 & \bar{u}+\bar{c} \\
0 & 2 \bar{u} & 0 \\
\bar{u}+\bar{c} & 0 & \bar{u}+\bar{c}
\end{array}\right] & \mathbf{L}_{\mathbf{s}}=\mathbf{B}^{+}=\frac{1}{2}\left[\begin{array}{ccc}
2 \bar{v} & 0 & 0 \\
0 & \bar{v}+\bar{c} & \bar{v}+\bar{c} \\
0 & \bar{v}+\bar{c} & \bar{v}+\bar{c}
\end{array}\right] \\
\mathbf{L}_{\mathbf{e}}=\mathbf{A}^{-}=\frac{1}{2}\left[\begin{array}{ccc}
\bar{u}-\bar{c} & 0 & \bar{c}-\bar{u} \\
0 & 0 & 0 \\
\bar{c}-\bar{u} & 0 & \bar{u}-\bar{c}
\end{array}\right] & \mathbf{L}_{\mathbf{n}}=\mathbf{B}^{-}=\frac{1}{2}\left[\begin{array}{ccc}
0 & 0 & 0 \\
0 & \bar{v}-\bar{c} & \bar{c}-\bar{v} \\
0 & \bar{c}-\bar{v} & \bar{v}-\bar{c}
\end{array}\right] .
\end{aligned}
$$

By using the operators (21), the boundary conditions of (18) becomes

$$
\begin{aligned}
& \mathbf{A}^{+} \mathbf{u}(0, y, t)=\mathbf{g}_{\mathbf{w}}(y, t) \\
& \mathbf{A}^{-} \mathbf{u}(1, y, t)=\mathbf{g}_{\mathbf{e}}(y, t) \\
& \mathbf{B}^{+} \mathbf{u}(x, 0, t)=\mathbf{g}_{\mathbf{s}}(x, t) \\
& \mathbf{B}^{-} \mathbf{u}(x, 1, t)=\mathbf{g}_{\mathbf{n}}(x, t) .
\end{aligned}
$$

$$
\begin{aligned}
& \|\mathbf{u}\|_{t}^{2}=\int_{x=0}^{1} \mathbf{g}_{\mathbf{s}}^{T}\left(\left[\mathbf{B}^{-1}\right]_{+}\right) \mathbf{g}_{\mathbf{s}} d x+\int_{x=0}^{1} \mathbf{u}(x, 0, t)^{T} \mathbf{B}^{-} \mathbf{u}(x, 0, t) d x \\
& -\int_{x=0}^{1} \mathbf{g}_{\mathbf{n}}^{T}\left(\left[\mathbf{B}^{-1}\right]_{-}\right) \mathbf{g}_{\mathbf{n}} d x-\int_{x=0}^{1} \mathbf{u}(x, 1, t)^{T} \mathbf{B}^{+} \mathbf{u}(x, 1, t) d x \\
& +\int_{y=0}^{1} \mathbf{g}_{\mathbf{w}}{ }^{T}\left(\left[\mathbf{A}^{-1}\right]_{+}\right) \mathbf{g}_{\mathbf{w}} d y+\int_{y=0}^{1} \mathbf{u}(0, y, t)^{T} \mathbf{A}^{-} \mathbf{u}(0, y, t) d x \\
& -\int_{y=0}^{1} \mathbf{g}_{\mathbf{e}}^{T}\left(\left[\mathbf{A}^{-1}\right]_{-}\right) \mathbf{g}_{\mathbf{e}} d y \quad-\int_{y=0}^{1} \mathbf{u}(1, y, t)^{T} \mathbf{A}^{+} \mathbf{u}(1, y, t) d x
\end{aligned}
$$

where $\left[\mathbf{A}^{-1}\right]_{ \pm}$and $\left[\mathbf{B}^{-1}\right]_{ \pm}$denotes the positive and negative part of the inverse of $\mathbf{A}$ and $\mathbf{B}$, respectively. Since $\mathbf{A}^{+}, \mathbf{B}^{+} \geq 0$ and $\mathbf{A}^{-}, \mathbf{B}^{-} \leq 0,(23)$ states that $\|\mathbf{u}\|_{t}$ is bounded by data. After integrating (23) in time, $\|\mathbf{u}\|$ will 
Table 1: The number of boundary conditions at each side of the unit square for the shallow water equations when considering the case $\bar{u}>0, \bar{v}>0$ and $\bar{u}^{2}+\bar{v}^{2}<\bar{c}$.

\begin{tabular}{c|c|c|c|c} 
Boundary & South & East & North & West \\
\hline Boundary conditions & 2 & 1 & 1 & 2
\end{tabular}

satisfy an estimate of the form (3). Since we have used the correct number of boundary conditions, we can summarize the results of this section in the following proposition.

Proposition 1. The problem (18) with $\bar{u}>0, \bar{v}>0$ and $\bar{u}^{2}+\bar{v}^{2}<\bar{c}$ is strongly well-posed if

$$
\mathbf{L}_{\mathbf{w}, \mathbf{e}}=\mathbf{A}^{ \pm}, \quad \mathbf{L}_{\mathbf{s}, \mathbf{n}}=\mathbf{B}^{ \pm}
$$

Remark 1. The number of boundary conditions at each boundary is equal to the rank of the corresponding boundary operator. For example, at the northern boundary, we have

$$
\mathbf{B}^{-} \mathbf{u}(x, 1, t)=\mathbf{g}_{n} \Longleftrightarrow(\bar{v}-\bar{c}) v^{\prime}+(\bar{c}-\bar{v}) \frac{g h^{\prime}}{\bar{c}}=g_{n}(x, t)
$$

where $g_{n}(x, t)$ is the boundary data. According to (24), one boundary condition is applied at $y=1$. The number of boundary conditions at each boundary is summarized in Table 1 .

Remark 2. With different signs of $\bar{u}, \bar{v}$, the boundary operators will change. For example, if $\bar{u}<0$, we need $\mathbf{L}_{\mathbf{w}}=\mathbf{A}^{-}, \mathbf{L}_{\mathbf{e}}=\mathbf{A}^{+}$.

\subsection{The semi-discrete formulation}

The semi-discrete approximation of (18) with the boundary operators (21) using the SBP-SAT technique is

$$
\begin{aligned}
\mathbf{v}_{t} & +\left(\mathbf{P}_{\mathbf{x}}{ }^{-1} \mathbf{Q}_{\mathbf{x}} \otimes \mathbf{I}_{\mathbf{y}} \otimes \mathbf{A}\right) \mathbf{v}+\left(\mathbf{I}_{\mathbf{x}} \otimes \mathbf{P}_{\mathbf{y}}{ }^{-1} \mathbf{Q}_{\mathbf{y}} \otimes \mathbf{B}\right) \mathbf{v}+\left(\mathbf{I}_{\mathbf{x}} \otimes \mathbf{I}_{\mathbf{y}} \otimes \mathbf{C}\right) \mathbf{v} \\
& =\left(\mathbf{P}_{\mathbf{x}}{ }^{-1} \mathbf{E}_{\mathbf{0}} \otimes \mathbf{P}_{\mathbf{y}}{ }^{-1} \otimes \mathbf{I}_{\mathbf{3}}\right) \boldsymbol{\Sigma}_{w}\left(\overline{\mathbf{A}}^{+} \mathbf{v}-\mathbf{g}_{\mathbf{w}}\right) \\
& +\left(\mathbf{P}_{\mathbf{x}}{ }^{-1} \mathbf{E}_{\mathbf{N}} \otimes \mathbf{P}_{\mathbf{y}}{ }^{-1} \otimes \mathbf{I}_{\mathbf{3}}\right) \boldsymbol{\Sigma}_{e}\left(\overline{\mathbf{A}}^{-} \mathbf{v}-\mathbf{g}_{\mathbf{e}}\right) \\
& +\left(\mathbf{P}_{\mathbf{x}}{ }^{-1} \otimes \mathbf{P}_{\mathbf{y}}{ }^{-1} \mathbf{E}_{\mathbf{0}} \otimes \mathbf{I}_{\mathbf{3}}\right) \boldsymbol{\Sigma}_{s}\left(\overline{\mathbf{B}}^{+} \mathbf{v}-\mathbf{g}_{\mathbf{s}}\right) \\
& +\left(\mathbf{P}_{\mathbf{x}}{ }^{-1} \otimes \mathbf{P}_{\mathbf{y}}{ }^{-1} \mathbf{E}_{\mathbf{N}} \otimes \mathbf{I}_{\mathbf{3}}\right) \boldsymbol{\Sigma}_{n}\left(\overline{\mathbf{B}}^{-} \mathbf{v}-\mathbf{g}_{\mathbf{n}}\right)
\end{aligned}
$$


where $\mathbf{P}_{\mathbf{x}, \mathbf{y}}$ are symmetric and positive definite, and $\mathbf{Q}_{\mathbf{x}, \mathbf{y}}$ satisfies the SBPproperty,

$$
\mathbf{Q}_{\mathbf{x}, \mathbf{y}}+\mathbf{Q}_{\mathbf{x}, \mathbf{y}}^{\mathbf{T}}=\mathbf{B}_{\mathbf{x}, \mathbf{y}}=-\mathbf{E}_{\mathbf{0}}+\mathbf{E}_{\mathbf{N}}=\operatorname{diag}(-1,0, \ldots, 0,1) .
$$

The subscripts on $\mathbf{Q}$ and $\mathbf{P}$ in 25 indicate what derivative that is approximated. In (25), $\mathbf{I}_{\mathbf{x}, \mathbf{y}}$ are identity matrices with the same size as $\mathbf{P}_{\mathbf{x}, \mathbf{y}}$, respectively, $\mathbf{I}_{\mathbf{3}}$ is a $3 \times 3$ identity matrix and $\mathbf{g}_{\mathbf{e}, \mathbf{w}, \mathbf{s}, \mathbf{n}}$ are the boundary data.

The symbol $\otimes$ in $(25)$ denotes the Kronecker product, which for two arbitrary matrices $\mathbf{C}$ and $\mathbf{D}$ is defined as

$$
\mathbf{C} \otimes \mathbf{D}=\left[\begin{array}{cccc}
c_{11} \mathbf{D} & a_{12} \mathbf{D} & \ldots & c_{1 m} \mathbf{D} \\
c_{21} \mathbf{D} & c_{22} \mathbf{D} & \ldots & \vdots \\
\vdots & \vdots & \ddots & \vdots \\
c_{n 1} \mathbf{D} & \ldots & \ldots & c_{n m} \mathbf{D}
\end{array}\right]
$$

where $c_{i j}$ are the elements of $\mathbf{C}$.

In (25), $\overline{\mathbf{A}}^{ \pm}=\mathbf{I}_{\mathbf{x}} \otimes \mathbf{I}_{\mathbf{y}} \otimes \mathbf{A}^{ \pm}$and $\overline{\mathbf{B}}^{ \pm}=\mathbf{I}_{\mathbf{x}} \otimes \mathbf{I}_{\mathbf{y}} \otimes \mathbf{B}^{ \pm}$and the matrices $\mathbf{E}_{\mathbf{0}, \mathbf{N}}$ are projection matrices, such that $\left(\mathbf{E}_{\mathbf{0}}\right)_{11}=\left(\mathbf{E}_{\mathbf{N}}\right)_{N N}=1$ and zero otherwise. The penalty matrices $\boldsymbol{\Sigma}_{w, s, e, n}$ will be determined such that stability is obtained and $\mathbf{g}_{\mathbf{w}, \mathbf{e}, \mathbf{s}, \mathbf{n}}$ are the boundary data.

By multiplying (25) with $\mathbf{v}^{T} \mathbf{P}_{\mathbf{x}} \otimes \mathbf{P}_{\mathbf{y}} \otimes \mathbf{I}_{\mathbf{3}}$ from the left and choosing $\boldsymbol{\Sigma}_{w, e}=\alpha_{1,2} \mathbf{I}_{\mathbf{x}} \otimes \mathbf{P}_{\mathbf{y}} \otimes \mathbf{I}_{\mathbf{3}}, \boldsymbol{\Sigma}_{s, n}=\alpha_{3,4} \mathbf{P}_{\mathbf{x}} \otimes \mathbf{I}_{\mathbf{y}} \otimes \mathbf{I}_{\mathbf{3}}$ with $\alpha_{1,3}=-1, \alpha_{2,4}=1$, one obtains

$$
\begin{aligned}
\left(\| \mathbf{v}||_{\mathbf{P}_{\mathbf{x}} \otimes \mathbf{P}_{\mathbf{y}} \otimes \mathbf{I}_{\mathbf{3}}}{ }^{2}\right)_{t}= & -\mathbf{v}^{T}\left(\mathbf{E}_{\mathbf{0}} \otimes \mathbf{P}_{\mathbf{y}} \otimes \mathbf{I}_{\mathbf{3}}\right) \overline{\mathbf{A}}^{+} \mathbf{v} \\
& +2 \mathbf{v}^{T}\left(\mathbf{E}_{\mathbf{0}} \otimes \mathbf{P}_{\mathbf{y}} \otimes \mathbf{I}_{\mathbf{3}}\right) \mathbf{g}_{\mathbf{w}}+\mathbf{v}^{T}\left(\mathbf{E}_{\mathbf{0}} \otimes \mathbf{P}_{\mathbf{y}} \otimes \mathbf{A}^{-}\right) \mathbf{v} \\
& +\mathbf{v}^{T}\left(\mathbf{E}_{\mathbf{N}} \otimes \mathbf{P}_{\mathbf{y}} \otimes \mathbf{I}_{\mathbf{3}}\right) \overline{\mathbf{A}}^{-} \mathbf{v} \\
& -2 \mathbf{v}^{T}\left(\mathbf{E}_{\mathbf{N}} \otimes \mathbf{P}_{\mathbf{y}} \otimes \mathbf{I}_{\mathbf{3}}\right) \mathbf{g}_{\mathbf{e}}-\mathbf{v}^{T}\left(\mathbf{E}_{\mathbf{N}} \otimes \mathbf{P}_{\mathbf{y}} \otimes \mathbf{A}^{+}\right) \mathbf{v} \\
& -\mathbf{v}^{T}\left(\mathbf{P}_{\mathbf{x}} \otimes \mathbf{E}_{\mathbf{0}} \otimes \mathbf{I}_{\mathbf{3}}\right) \overline{\mathbf{B}}^{+} \mathbf{v} \\
& +2 \mathbf{v}^{T}\left(\mathbf{P}_{\mathbf{x}} \otimes \mathbf{E}_{\mathbf{0}} \otimes \mathbf{I}_{\mathbf{3}}\right) \mathbf{g}_{\mathbf{s}}+\mathbf{v}^{T}\left(\mathbf{P}_{\mathbf{x}} \otimes \mathbf{E}_{\mathbf{0}} \otimes \mathbf{B}^{-}\right) \mathbf{v} \\
& +\mathbf{v}^{T}\left(\mathbf{P}_{\mathbf{x}} \otimes \mathbf{E}_{\mathbf{N}} \otimes \mathbf{I}_{\mathbf{3}}\right) \overline{\mathbf{B}}^{-} \mathbf{v} \\
& -2 \mathbf{v}^{T}\left(\mathbf{P}_{\mathbf{x}} \otimes \mathbf{E}_{\mathbf{0}} \otimes \mathbf{I}_{\mathbf{4}}\right) \mathbf{g}_{\mathbf{n}}-\mathbf{v}^{T}\left(\mathbf{P}_{\mathbf{x}} \otimes \mathbf{E}_{\mathbf{N}} \otimes \mathbf{B}^{+}\right) \mathbf{v} .
\end{aligned}
$$


continuous case. By completing the squares, (26) finally becomes

$$
\begin{aligned}
& \left(\|\mathbf{v}\|_{\mathbf{P}_{\mathbf{x}} \otimes \mathbf{P}_{\mathbf{y}} \otimes \mathbf{I}_{\mathbf{3}}}{ }^{2}\right)_{t}= \\
& +\mathbf{g}_{\mathbf{w}}{ }^{T}\left(\mathbf{E}_{\mathbf{0}} \otimes \mathbf{P}_{\mathbf{y}} \otimes\left[\mathbf{A}^{-1}\right]_{+}\right) \mathbf{g}_{\mathbf{w}}+\mathbf{v}^{T}\left(\mathbf{E}_{\mathbf{0}} \otimes \mathbf{P}_{\mathbf{y}} \otimes \mathbf{A}^{-}\right) \mathbf{v} \\
& -\mathbf{g}_{\mathbf{e}}^{T}\left(\mathbf{E}_{\mathbf{N}} \otimes \mathbf{P}_{\mathbf{y}} \otimes\left[\mathbf{A}^{-1}\right]_{-}\right) \mathbf{g}_{\mathbf{e}}-\mathbf{v}^{T}\left(\mathbf{E}_{\mathbf{N}} \otimes \mathbf{P}_{\mathbf{y}} \otimes \mathbf{A}^{+}\right) \mathbf{v} \\
& +\mathbf{g}_{\mathbf{s}}{ }^{T}\left(\mathbf{P}_{\mathbf{x}} \otimes \mathbf{E}_{\mathbf{0}} \otimes\left[\mathbf{B}^{-1}\right]_{+}\right) \mathbf{g}_{\mathbf{s}}+\mathbf{v}^{T}\left(\mathbf{P}_{\mathbf{x}} \otimes \mathbf{E}_{\mathbf{0}} \otimes \mathbf{B}^{-}\right) \mathbf{v} \\
& -\mathbf{g}_{\mathbf{n}}{ }^{T}\left(\mathbf{P}_{\mathbf{x}} \otimes \mathbf{E}_{\mathbf{N}} \otimes\left[\mathbf{B}^{-1}\right]_{-}\right) \mathbf{g}_{\mathbf{n}}-\mathbf{v}^{T}\left(\mathbf{P}_{\mathbf{x}} \otimes \mathbf{E}_{\mathbf{N}} \otimes \mathbf{B}^{+}\right) \mathbf{v} \\
& -\left(\overline{\mathbf{A}}^{+} \mathbf{v}-\mathbf{g}_{\mathbf{w}}\right)^{T}\left(\mathbf{E}_{\mathbf{0}} \otimes \mathbf{P}_{\mathbf{y}} \otimes\left[\mathbf{A}^{-1}\right]_{+}\right)\left(\overline{\mathbf{A}}^{+} \mathbf{v}-\mathbf{g}_{\mathbf{w}}\right) \\
& +\left(\overline{\mathbf{A}}^{-} \mathbf{v}-\mathbf{g}_{\mathbf{e}}\right)^{T}\left(\mathbf{E}_{\mathbf{N}} \otimes \mathbf{P}_{\mathbf{y}} \otimes\left[\mathbf{A}^{-1}\right]_{-}\right)\left(\overline{\mathbf{A}}^{-} \mathbf{v}-\mathbf{g}_{\mathbf{e}}\right) \\
& -\left(\overline{\mathbf{B}}^{+} \mathbf{v}-\mathbf{g}_{\mathbf{s}}\right)^{T}\left(\mathbf{P}_{\mathbf{x}} \otimes \mathbf{E}_{\mathbf{0}} \otimes\left[\mathbf{B}^{-1}\right]_{+}\right)\left(\overline{\mathbf{B}}^{+} \mathbf{v}-\mathbf{g}_{\mathbf{s}}\right) \\
& +\left(\overline{\mathbf{B}}^{-} \mathbf{v}-\mathbf{g}_{\mathbf{n}}\right)^{T}\left(\mathbf{P}_{\mathbf{x}} \otimes \mathbf{E}_{\mathbf{N}} \otimes\left[\mathbf{B}^{-1}\right]_{-}\right)\left(\overline{\mathbf{B}}^{-} \mathbf{v}-\mathbf{g}_{\mathbf{n}}\right) .
\end{aligned}
$$

In $(27),\left[\mathbf{A}^{-1}\right]_{ \pm}$and $\left[\mathbf{B}^{-1}\right]_{ \pm}$refers to the negative and positive parts of the inverses of $\mathbf{A}$ and $\mathbf{B}$, respectively.

The matrices $\left[\mathbf{A}^{-1}\right]_{+},\left[\mathbf{B}^{-1}\right]_{+}, \mathbf{A}^{+}$and $\mathbf{B}^{+}$are all positive semi definite, while the matrices $\left[\mathbf{A}^{-1}\right]_{-},\left[\mathbf{B}^{-1}\right]_{-}, \mathbf{A}^{-}$and $\mathbf{B}^{-}$are all negative semi definite. This implies that the only terms that are non-negative are the ones containing data. Hence, the growth of $\mathbf{v}$ is bounded by applied data. After timeintegration, (27) will satisfy an estimate of the form (6), and (25) is therefore strongly stable. Note that the terms $\left(\mathbf{g}_{\mathbf{w}, \mathbf{e}}\right)^{T}\left(\mathbf{E}_{\mathbf{0 , N}} \otimes \mathbf{P}_{\mathbf{y}} \otimes\left[\mathbf{A}^{-1}\right]_{ \pm}\right) \mathbf{g}_{\mathbf{w}, \mathbf{e}}$ and the terms $\left(\mathbf{g}_{\mathbf{s}, \mathbf{n}}\right)^{T}\left(\mathbf{P}_{\mathbf{x}} \otimes \mathbf{E}_{\mathbf{0}, \mathbf{N}} \otimes\left[\mathbf{A}^{-1}\right]_{ \pm}\right) \mathbf{g}_{\mathbf{s}, \mathbf{n}}$ in 27$)$ mimics the integrals in (23) containing the boundary data, and $\mathbf{v}^{T}\left(\mathbf{E}_{\mathbf{0}, \mathbf{N}} \otimes \mathbf{P}_{\mathbf{y}} \otimes \mathbf{A}^{ \pm}\right) \mathbf{v}$ and $\mathbf{v}^{T}\left(\mathbf{P}_{\mathbf{x}} \otimes \mathbf{E}_{\mathbf{0}, \mathbf{N}} \otimes \mathbf{B}^{ \pm}\right) \mathbf{v}$ mimics the remaining integrals. Hence, (27) mimics the continuous energy estimate, given by (23), if the small damping terms are neglected.

We summarize the results of this section in the following proposition.

Proposition 2. The semi-discrete approximation (25) with

$$
\begin{aligned}
& \boldsymbol{\Sigma}_{w, e}=\alpha_{1,2} \mathbf{I}_{\mathbf{x}} \otimes \mathbf{P}_{\mathbf{y}} \otimes \mathbf{I}_{\mathbf{3}} \\
& \boldsymbol{\Sigma}_{s, n}=\alpha_{3,4} \mathbf{P}_{\mathbf{x}} \otimes \mathbf{I}_{\mathbf{y}} \otimes \mathbf{I}_{\mathbf{3}}
\end{aligned}
$$

is strongly stable if $\alpha_{1,3}=-1$ and $\alpha_{2,4}=1$.

\subsection{The multiple penalty technique}

Next, we move on to the MPT where additional SAT's are applied at several grid points in the domain. We denote the set of these grid points 
$\Omega_{M}$. To ease the derivations, we consider the error equation corresponding to (25) with additional penalty terms.

By inserting the analytic solution, $\mathbf{u}$, into $(25)$ and subtracting the result from (25) with the numerical solution $\mathbf{v}$, one obtains the error equation

$$
\begin{aligned}
\mathbf{e}_{t} & +\left(\mathbf{P}_{\mathbf{x}}{ }^{-1} \mathbf{Q}_{\mathbf{x}} \otimes \mathbf{I}_{\mathbf{y}} \otimes \mathbf{A}\right) \mathbf{e}+\left(\mathbf{I}_{\mathbf{x}} \otimes \mathbf{P}_{\mathbf{y}}{ }^{-1} \mathbf{Q}_{\mathbf{y}} \otimes \mathbf{B}\right) \mathbf{e}= \\
& -\left(\mathbf{P}_{\mathbf{x}}{ }^{-1} \mathbf{E}_{\mathbf{0}} \otimes \mathbf{I}_{\mathbf{y}} \otimes \mathbf{I}_{\mathbf{3}}\right) \overline{\mathbf{A}}^{+} \mathbf{e} \\
& +\left(\mathbf{P}_{\mathbf{x}}{ }^{-1} \mathbf{E}_{\mathbf{N}} \otimes \mathbf{I}_{\mathbf{y}} \otimes \mathbf{I}_{\mathbf{3}}\right) \overline{\mathbf{A}}^{-} \mathbf{e} \\
& -\left(\mathbf{I}_{\mathbf{x}} \otimes \mathbf{P}_{\mathbf{y}}{ }^{-1} \mathbf{E}_{\mathbf{0}} \otimes \mathbf{I}_{\mathbf{3}}\right) \overline{\mathbf{B}}^{+} \mathbf{e} \\
& +\left(\mathbf{I}_{\mathbf{x}} \otimes \mathbf{P}_{\mathbf{y}}{ }^{-1} \mathbf{E}_{\mathbf{N}} \otimes \mathbf{I}_{\mathbf{3}}\right) \overline{\mathbf{B}}^{-} \mathbf{e}+ \\
& +\sum_{i \in \Omega_{M}}\left(\mathbf{P}_{\mathbf{x}}{ }^{-1} \mathbf{E}_{\mathbf{i}} \otimes \mathbf{I}_{\mathbf{y}} \otimes \mathbf{\Sigma}^{\mathbf{i}}\right) \mathbf{L}_{\mathbf{i}}^{\mathbf{M P}} \mathbf{e}+\mathbf{T e}
\end{aligned}
$$

where $\mathbf{e}=\mathbf{v}-\mathbf{u}$ is the error and Te is the truncation error. The terms $\left(\mathbf{P}_{\mathbf{x}}{ }^{-1} \mathbf{E}_{\mathbf{i}} \otimes \mathbf{I}_{\mathbf{y}} \otimes \boldsymbol{\Sigma}^{\mathbf{i}}\right) \mathbf{L}_{\mathbf{i}}^{\mathbf{M P}} \mathbf{e}$ are the MPT's in $\Omega_{M}$. The elements of $\mathbf{E}_{\mathbf{i}}$ are $\left(\mathbf{E}_{\mathbf{i}}\right)_{i i}=1$ and zero otherwise. In $(28)$, we have used the penalty matrices in Proposition 2 at the physical boundaries. The operators $\mathbf{L}_{\mathbf{i}}^{\mathbf{M P}}$ are arbitrary boundary operators in $\Omega_{M}$, and $\Sigma^{i}$ are penalty matrices to be determined such that the scheme is stable. To ease the derivations, we neglect the truncation error Te. It can be shown that neglecting the truncation error will not affect the following results.

By multiplying (28) with $\mathbf{e}^{T}\left(\mathbf{P}_{\mathbf{x}} \otimes \mathbf{P}_{\mathbf{y}} \otimes \mathbf{I}_{\mathbf{3}}\right)$ from the left and adding the transpose of the outcome to itself, one arrives at

$$
\|\mathbf{e}\|_{t}^{2} \leq \sum_{i \in \Omega_{M}} \mathbf{e}^{\mathbf{T}} \mathbf{E}_{\mathbf{i}} \otimes \mathbf{P}_{\mathbf{y}} \otimes\left[\Sigma^{\mathrm{i}} \mathbf{L}_{\mathbf{i}}^{\mathbf{M P}}+\left(\Sigma^{\mathrm{i}} \mathbf{L}_{\mathbf{i}}^{\mathbf{M P}}\right)^{\mathbf{T}}\right] \mathbf{e}
$$

since the remaining terms only contribute with a decay of energy, as concluded in section 4.2. Every choice of $\Sigma^{\mathbf{i}}$ that makes the right-hand side of (29) non-positive results in a decay of the error, and a stable scheme. Since $\mathbf{P}_{\mathbf{y}}$ is positive definite and $\mathbf{E}_{\mathbf{i}}$ is positive semi-definite, the right-hand side of 29] is non-positive if we choose $\boldsymbol{\Sigma}^{\mathbf{i}}$ such that $\boldsymbol{\Sigma}^{\mathbf{i}} \mathbf{L}_{\mathbf{i}}^{\mathbf{M P}}+\left(\boldsymbol{\Sigma}^{\mathbf{i}} \mathbf{L}_{\mathbf{i}}^{\mathbf{M P}}\right)^{\mathbf{T}} \leq 0$.

We summarize the results in the following proposition.

Proposition 3. The MPT applied in (28) preserves stability if

$$
\Sigma^{\mathrm{i}} \mathbf{L}_{\mathbf{i}}^{\mathrm{MP}}+\left(\Sigma^{\mathrm{i}} \mathbf{L}_{\mathbf{i}}^{\mathrm{MP}}\right)^{\mathbf{T}} \leq 0
$$

where $\mathbf{L}_{\mathbf{i}}^{\mathbf{M P}}$ are arbitrarily chosen boundary operators. 
In this work, we apply the MPT close to the boundaries and use the operators $\mathbf{L}_{\mathbf{w}, \mathbf{e}}^{\mathbf{M P}}=\overline{\mathbf{A}}^{ \pm}$and $\mathbf{L}_{\mathbf{s}, \mathbf{n}}^{\mathbf{M P}}=\overline{\mathbf{B}}^{ \pm}$, where $w, e, s, n$ denotes the boundary where the MPT is applied. The penalty matrices are $\boldsymbol{\Sigma}_{e, w}^{i}= \pm \mathbf{I}_{\mathbf{3}}$ and $\boldsymbol{\Sigma}_{n, s}^{i}= \pm \mathbf{I}_{\mathbf{3}}$. One can easily confirm that these choices satisfy (30).

In (28), we have assumed that data is available in the domain $\Omega_{M}$. When considering local area models, as illustrated in Figure 3 , the data in $\Omega_{M}$ is obtained by interpolating the results from a global calculation on a coarse mesh. In the general case, however, the required data can be obtained in several ways, for example by measurements.

Remark 3. It is easy to show that (30) is satisfied if $\boldsymbol{\Sigma}^{\mathbf{i}}=\gamma\left(\mathbf{L}_{\mathbf{i}}^{\mathbf{M P}}\right)^{\mathbf{T}}$ for any constant $\gamma \leq 0$. Hence, one can always find penalty matrices that fulfills Proposition 3 .

Remark 4. As illustrated in section 3 and (29), the MPT contributes with additional damping terms. This accelerates the rate of convergence, even at grid points where no data is available.

\section{Numerical results}

In our first steady-state test case, we consider (18) with the reference state $\bar{u}=\bar{v}=1 \mathrm{~ms}^{-1}, \bar{c}=2 \mathrm{~ms}^{-1}$ and $\mathrm{g}=9.82 \mathrm{~ms}^{-2}$. The following results are obtained for sub-critical flows. Similar results are obtained for super-critical flows, as illustrated in Appendix A.

The local area calculation is modeled by considering a standing wave of the form $\mathbf{u}(x, y)=\sin (2 \pi x)[1,1,1]^{T}$ that we consider coming from a global calculation. This solution is obtained by choosing $\mathbf{F}(x, y, t)$ and $\mathbf{g}_{\mathbf{w}, \mathbf{e}, \mathbf{s}, \mathbf{n}}$ accordingly in (18) and adding random errors to it. At $t=0$, we consider the wave to be poorly resolved, as shown to the right in Figure 4 , and we wish to get a better resolution by applying the MPT close to the boundaries.

Figures 547 show the deviation from the steady-state solution using $N_{M P}=$ $r N$ ( $r$ is a number between zero and one) additional penalty terms in the vicinity of each boundary with $N=20,40,80$ grid points in each space direction. At grid point $i, j$ in the penalty domains, we use data from the steadystate solution, i.e. $g\left(x_{i}, y_{j}\right)=\sin \left(2 \pi x_{i}\right)[1,1,1]^{T}$, when applying the MPT. For the spatial discretization, we use a third order accurate SBP scheme. The lateral boundary operators used are $\mathbf{L}_{\mathbf{w}, \mathbf{e}}^{\mathbf{M P}}=\overline{\mathbf{A}}^{ \pm}$and $\mathbf{L}_{\mathbf{s}, \mathbf{n}}^{\mathbf{M P}}=\overline{\mathbf{B}}^{ \pm}$where $w, e, s, n$ denotes the boundary where the MPT is applied. We use the penalty 

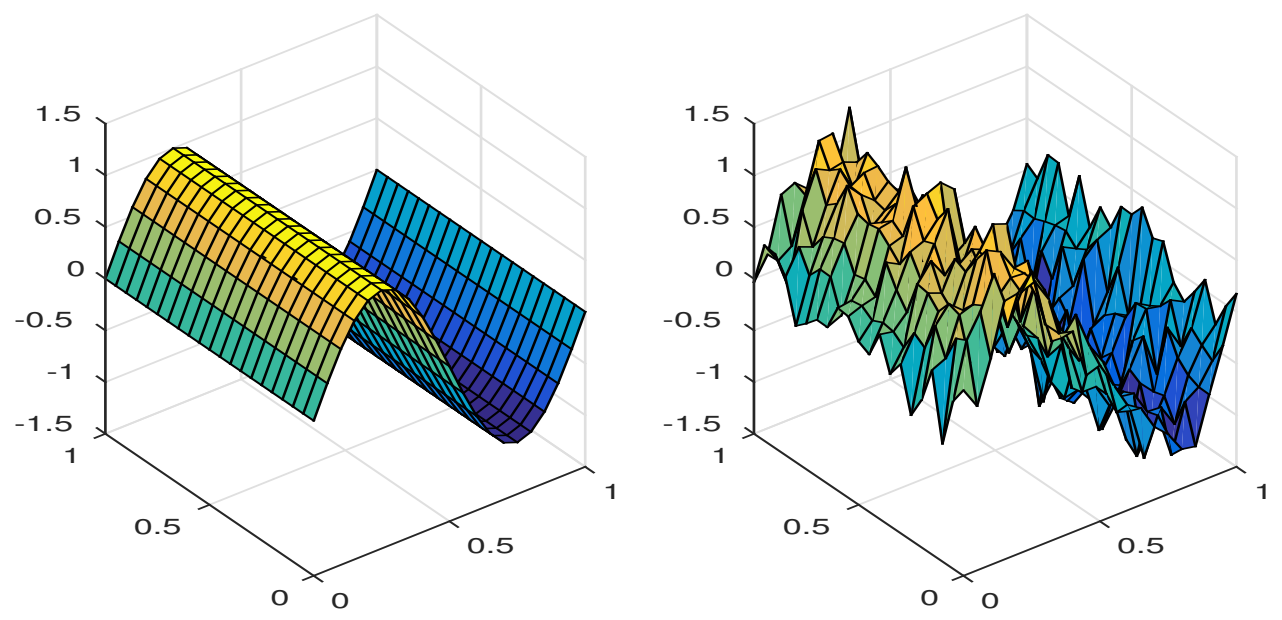

Figure 4: Right: the unresolved solution at $t=0$ modeled by adding random errors at each grid point. Left: the solution at steady-state.

matrices $\boldsymbol{\Sigma}_{e, w}^{i}= \pm \mathbf{I}_{\mathbf{3}}$ and $\boldsymbol{\Sigma}_{n, s}^{i}= \pm \mathbf{I}_{\mathbf{3}}$ to preserve stability. As one can see, the error levels are greatly reduced when the MPT is applied.

\subsection{Time-dependent solutions}

For the second time-dependent test case, we let the disturbance in height, $h^{\prime}$, vary as an oscillating pulse of the form

$$
h^{\prime}=\exp \left(-10 r^{2}\right) \sin \left[2 \pi\left(r^{2}-t\right)\right]
$$

where $r^{2}=(x-0.5)^{2}+(y-0.5)^{2}$. The oscillating pulse is manufactured by choosing the boundary data and $\mathbf{F}(x, y, t)$ in $(18)$ accordingly. We apply the MPT at $r=N_{M P} / N$ additional grid points in the vicinity of each boundary. The applied data in the penalty regions is $g\left(x_{i}, y_{j}\right)=h^{\prime}\left(x_{i}, y_{j}\right)=$ $\exp \left(-10 r_{i, j}^{2}\right) \sin \left[2 \pi\left(r_{i, j}^{2}-t\right)\right]$, where $r_{i, j}^{2}=\left(x_{i}-0.5\right)^{2}+\left(y_{j}-0.5\right)^{2}$. The simulation starts from the initial condition $\mathbf{u}=0$, and is then integrated in time using a fourth order explicit Runge-Kutta method. The solution at different time levels is displayed in Figure 8, and the deviation (or error) from the exact solution as a function of time is shown in Figure 9-11. As one can see, the MPT speeds up the rate of convergence also for time-dependent problems. 


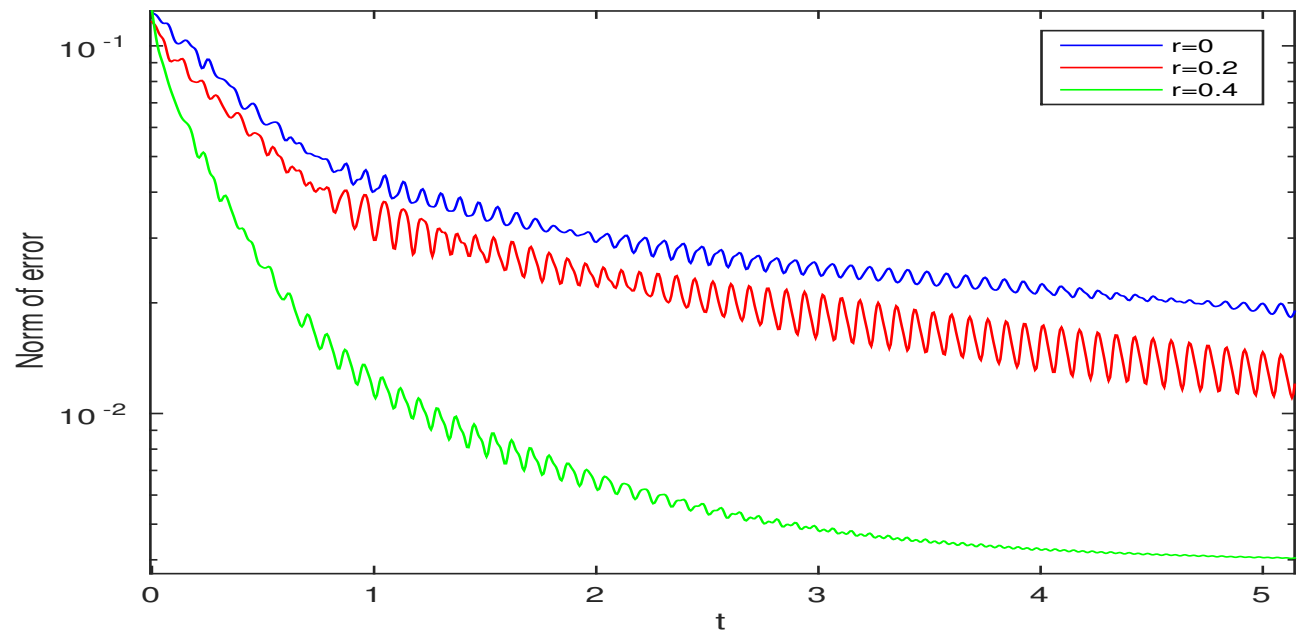

Figure 5: The error as function of time for the shallow water equations. $N=20$ and $r=N_{M P} / N$.

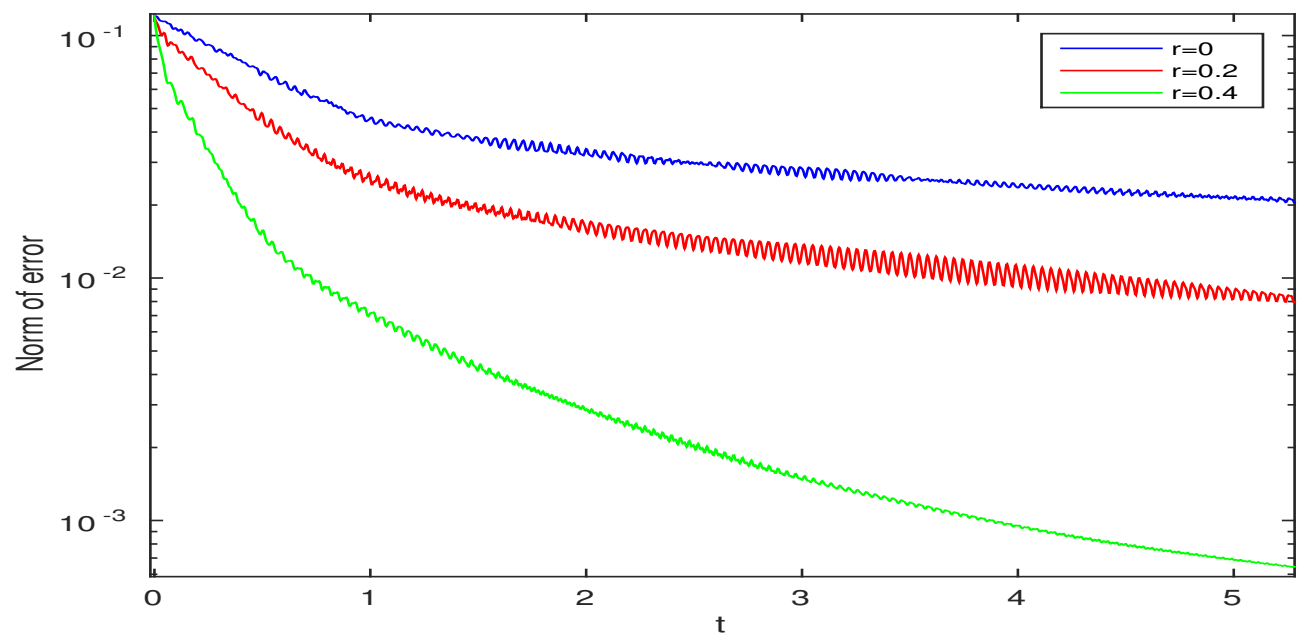

Figure 6: The error as function of time for the shallow water equations. $N=40$ and $r=N_{M P} / N$. 


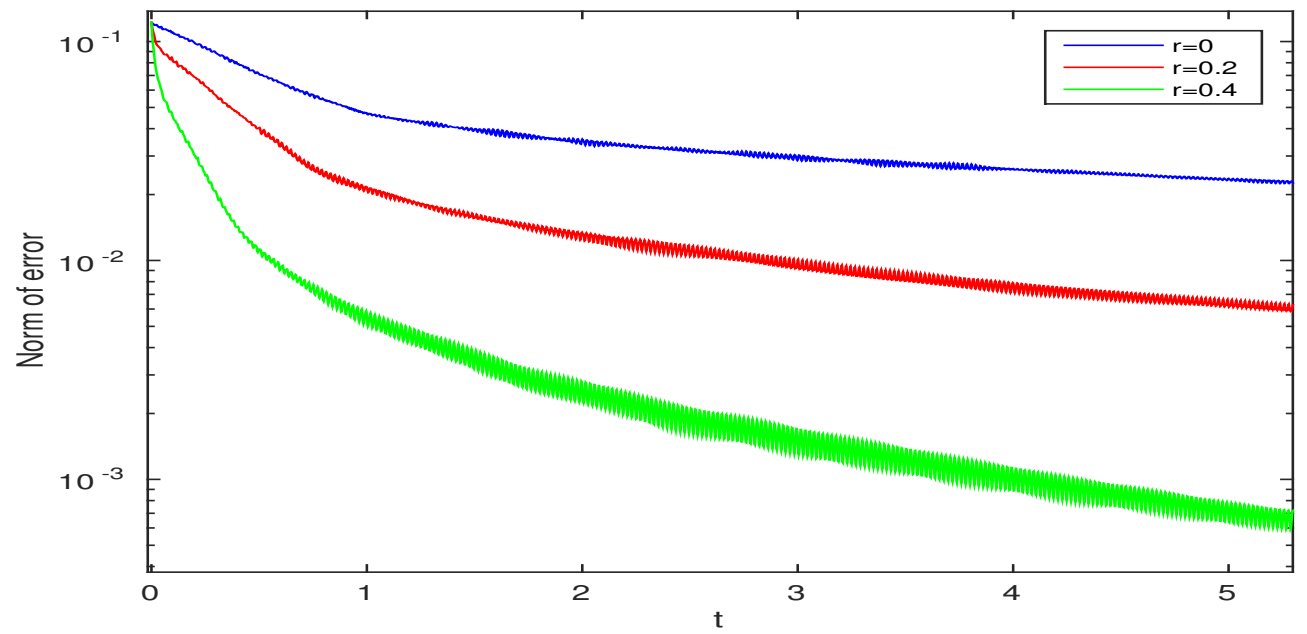

Figure 7: The error as function of time for the shallow water equations. $N=80$ and $r=N_{M P} / N$.
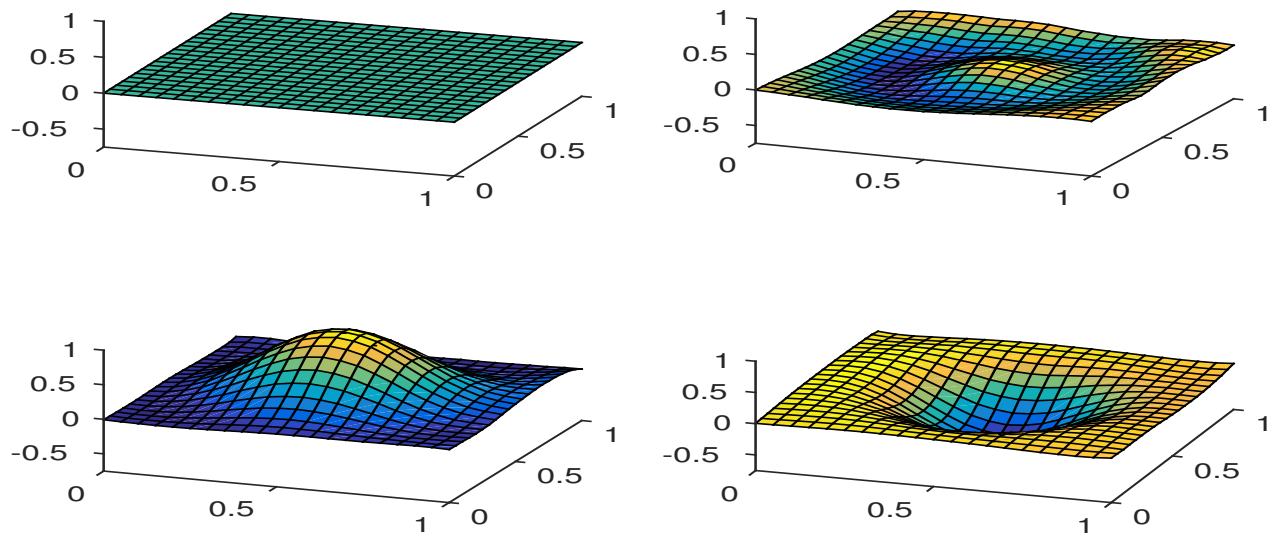

Figure 8: The computed time-dependent solution of $h^{\prime}$ at different time levels. Upper left: $t=0$. Upper right: $t=0.5$. Lower left: $t=0.75$. Lower right: $t=1.15$. 


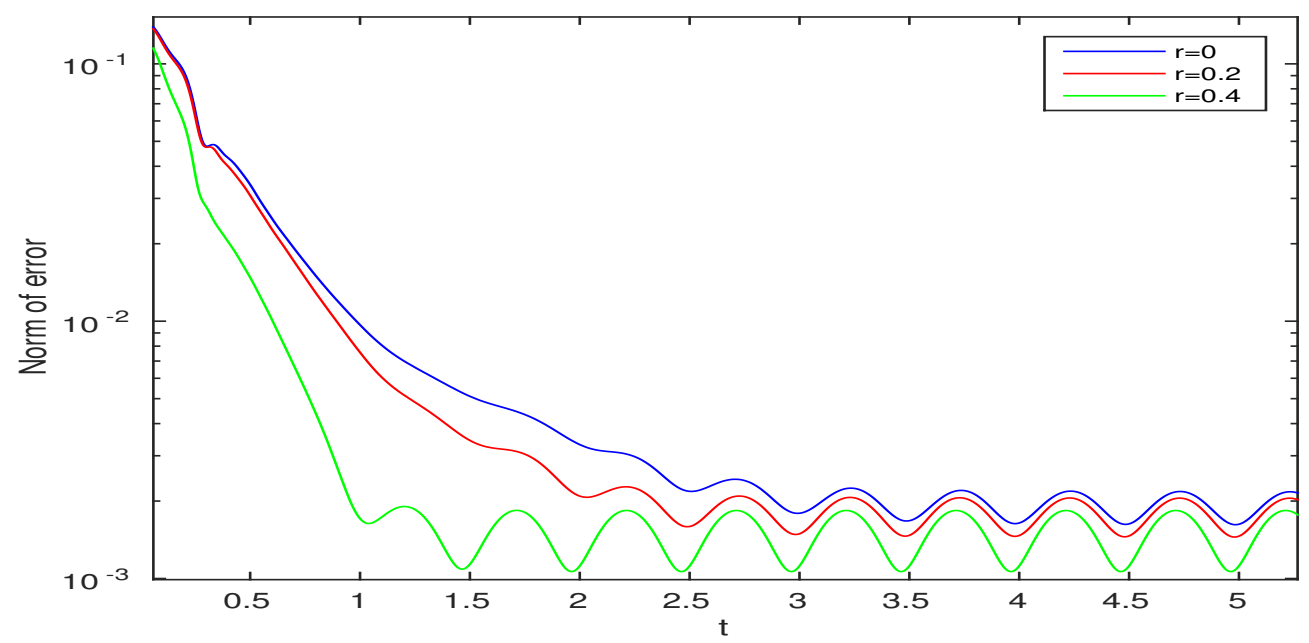

Figure 9: The error of $h^{\prime}$ as function of time for the shallow water equations using timedependent boundary data. $N=20$ and $r=N_{M P} / N$.

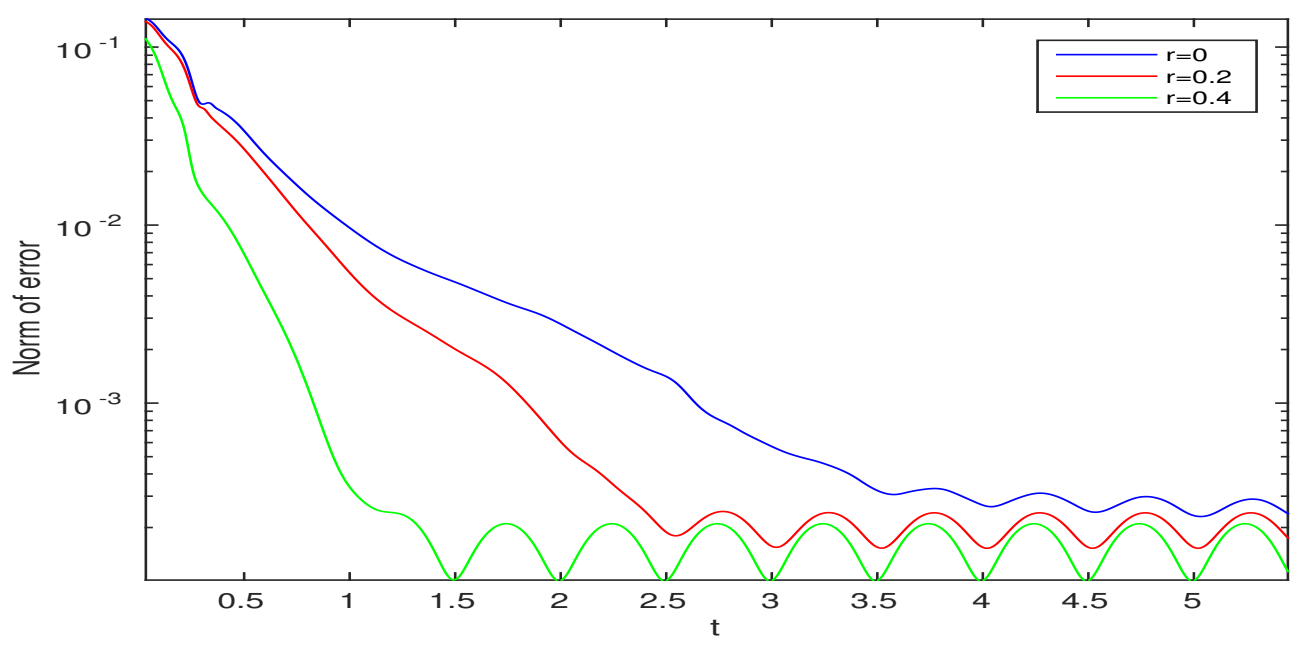

Figure 10: The error of $h^{\prime}$ as function of time for the shallow water equations using time-dependent boundary data. $N=40$ and $r=N_{M P} / N$. 


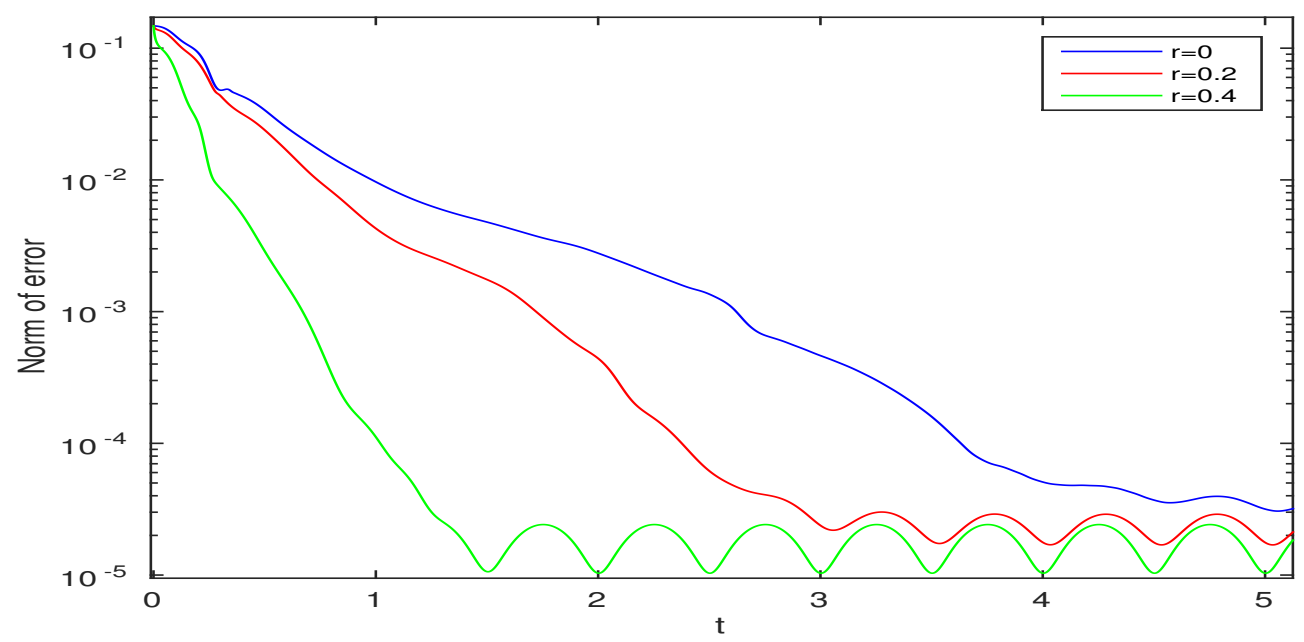

Figure 11: The error of $h^{\prime}$ as function of time for the shallow water equations using time-dependent boundary data. $N=80$ and $r=N_{M P} / N$.

Remark 5. All numerical experiments in this work are performed using a third order accurate SBP scheme. A higher order of accuracy has only a small effect on the rate of convergence. The increased rate of convergence is mainly due to the additional damping terms in (29).

\section{Summary and conclusions}

A new lateral boundary procedure (MPT) similar to the one introduced by Davies (1976) has been applied to the linearized shallow water equations. The technique builds on a generalization of the SBP-SAT technique.

If well-posed boundary conditions are available, the shallow water equations can be discretized using the SBP-SAT technique, such that the resulting scheme is stable. It was shown how additional SAT's can be applied inside the domain, while maintaining stability. The form of the additional SAT's is completely general, and stability can always be obtained by appropriate choices of penalty matrices. All theoretical results of this paper are also general, and can easily be extended to other partial differential equations, such as the Euler and Navier-Stokes equations.

The technique is straight-forward to implement, very general and stability 
is easily shown by energy methods. Existing techniques building on Davies relaxation can easily be made stable using the MPT technique.

The additional penalty terms increases the rate of convergence to the correct solution for both steady-state and time-dependent problems. In the cases considered in this work, the increase of the rate of convergence is significant.

\section{Acknowledgments}

This project was funded by the Swedish e-science Research Center (SeRC). The funding source had no involvement in the study design, the collection and analysis of data or in writing and submitting this article.

\section{Appendix A.}

Consider (18) with the reference state $\bar{u}=\bar{v}=3 / 2 \mathrm{~ms}^{-1}, \bar{c}=2 \mathrm{~ms}^{-1}$ and $g=9.82 \mathrm{~ms}^{-2}$, such that $\bar{u}^{2}+\bar{v}^{2}>\bar{c}^{2}$. As in Section 5, we construct a steady-state solution $\mathbf{u}(x, y)=\sin (2 \pi x)[1,1,1]^{T}$ by choosing the forcing function and boundary data accordingly. The MPT is applied at $N_{M P}=r N$ grid points in the vicinity of each boundary. A SBP scheme with third order overall accuracy is used for the space discretization and a fourth order implicit Runge-Kutta is used to integrate in time. The simulation starts from a poorly resolved wave, as illustrated in Figure 4, and the error as function of time is displayed in Figure A.12. As one can see, the results are similar to the ones obtained in Section 5, where sub-critical flows were considered.

\section{References}

Birchfield, G.E., 1960. Numerical prediction of hurricane movement with 口 the use of a fine grid. Journal of Meteorology 17, 406-414. doi:10.1175/ 1520-0469(1960)017<0406:NPOHMW>2.0.C0;2.

Carpenter, M., Gottlieb, D., Abarbanel, S., 1994. Time-stable boundary conditions for finite-difference schemes solving hyperbolic systems: methodology and application to high-order compact schemes. Journal of Computational Physics 111, 220-236. doi:10.1006/jcph.1994.1057.

Carpenter, M., Nordström, J., Gottlieb, D., 1999. A stable and conservative interface treatment of arbitrary spatial accuracy. Journal of Computational Physics 148, 341-365. doi:10.1006/jcph.1998.6114. 


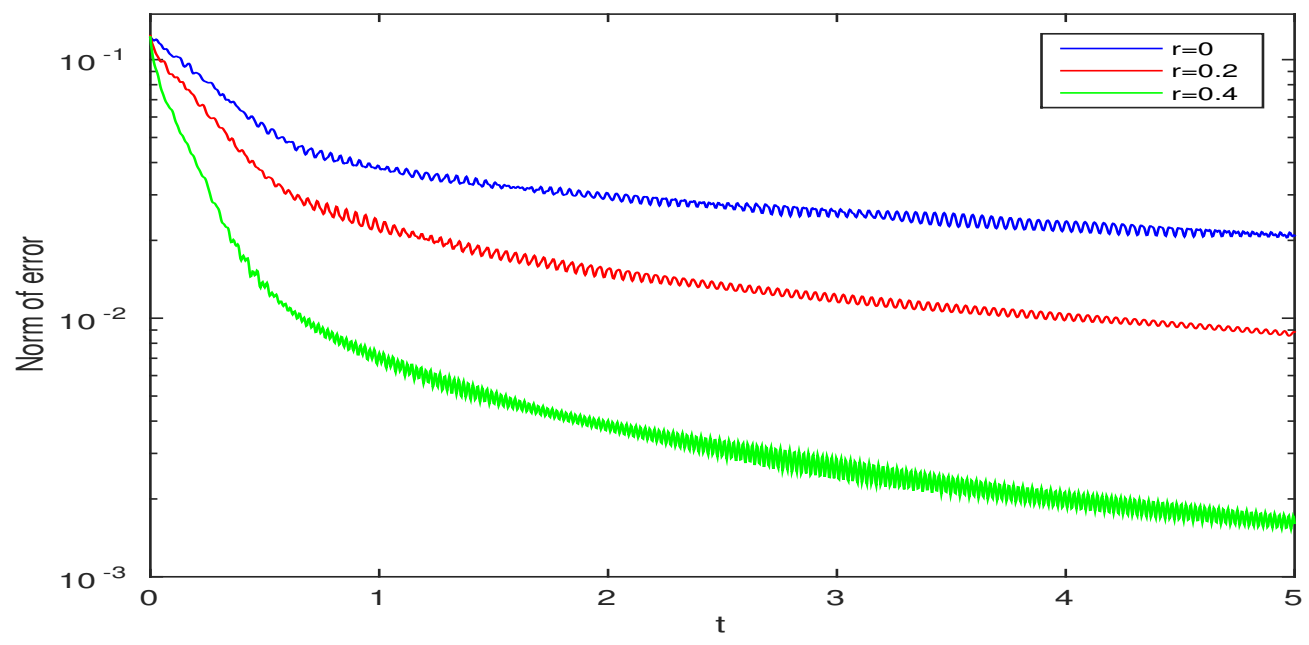

Figure A.12: The error as function of time for the shallow water equations with supercritical flows. $N=40$ and $r=N_{M P} / N$.

Davies, H., 1983. Limitations of some common lateral boundary schemes used in regional NWP models. Monthly Weather review 111, 1002-1012. doi:10.1175/1520-0493(1983)111>1002:LOSCLB>2.0.CO:2.

Davies, H.C., 1976. A lateral boundary formulation for multi-level prediction models. Quart. J. Roy. Meteor. Soc. 102, 406-418. doi:10.1002/qj . 49710243210.

Erickson, B., Nordström, J., 2014. Stable, high order accurate adaptive schemes for long time, highly intermittent geophysics problems. Journal of Computational and Applied Mathematics 271, 328-338. doi:10.1016/ j.cam.2014.04.019.

Ghader, S., Nordström, J., 2014. Revisiting well-posed boundary conditions for the shallow water equations. Dynamics of Atmospheres and Oceans 66, 1-9. doi:10.1016/j.dynatmoce.2014.01.002.

Harrison, E.J., Elsberry, R.L., 1972. A method for incorporating nested finite grids in the solution of systems of geophysical equations. Journal of Atmospheric Sciences 29, 1235-1245. doi:10.1175/1520-0469(1972) $029<1235$ : AMFINF>2.0.CO;2. 
Hill, G.E., 1968. Grid telescoping in numerical weather prediction. Journal of Applied Meteorology 7, 29-38. doi:10.1175/1520-0450(1968)007<0029: GTINWP $>2.0 . \mathrm{CO} ; 2$.

Lehmann, R., 1993. On the choice of relaxation coefficients for Davies lateral boundary scheme for regional weather prediction. Meteorology and Atmospheric Physics 52, 1-14. doi:10.1007/BF01025749.

Lindström, J., Nordström, J., 2010. A stable and high-order accurate conjugate heat transfer problem. Journal of Computational Physics 229, 54405456. doi:10.1016/j.jcp.2010.04.010.

Mattsson, K., Nordström, J., 2004. Summation by parts for finite difference approximations of second order derivatives. Journal of Computational Physics 199, 503-540. doi:10.1016/j.jcp.2004.03.001.

Nordström, J., Abbas, Q., Erickson, B., Frenander, H., 2014. A flexible boundary procedure for hyperbolic problems: multiple penalty terms applied in a domain. Communications in Computational Physics 16, 541-570. doi:10.4208/cicp.020313.120314a.

Nordström, J., Gong, J., Weide, E., Svärd, M., 2009a. A stable and conservative high order multiblock method for the compressible NavierStokes equations. Journal of Computational Physics 228, 9020-9035. doi:10.1016/j.jcp.2009.09.005.

Nordström, J., Ham, F., Shoeybi, M., Weide, E., Svärd, M., Mattsson, K., Iaccarino, G., Gong, J., 2009b. A hybrid method for unsteady inviscid fluid flow. Computers and Fluids 38, 875-882. doi:10.1016/j.compfluid. 2008.09.010.

Strand, B., 1994. Summation by parts for finite difference approximations 7or $\frac{d}{d x}$. Journal of Computational Physics 110, 47-67. doi:10.1006/jcph. 1994.1005.

Svärd, M., Nordström, J., 2014. Review of summation-by-parts schemes for initial-boundary-value problems. Journal of Computational Physics 268, 17-30. doi:10.1016/j.jcp.2014.02.031. 
${ }_{427}$ Williamson, D.L., Browning, G.L., 1974. Formulation of the lateral boundary ${ }_{428}$ conditions for the NCAR limited-area model. Journal of Applied Mete429 orology $13,8-16$. doi $10.1175 / 1520-0450$ (1974) 013<0008: FOTLBC>2. 0 . ${ }_{430} \mathrm{CO} ; 2$. 